\title{
Phononic Band Gaps in One-dimensional Phononic Crystals with Nanoscale Periodic Corrugations at Interfaces. FDTD and PWM Simulations
}

\author{
Przemyslaw Nowak, Maciej Krawczyk \\ Surface Physics Division, Faculty of Physics, Adam Mickiewicz University \\ ul. Umultowska 85, 61-614 Poznań, Poland \\ e-mail:pnowak@atrom.au
}

(Received: 31 December 2009; revised: 26 February 2010; accepted: 10 March 2010; published online: 29 April 2010))

\begin{abstract}
We present and apply two complementary calculation methods used in phononic crystal studies: the finite difference time domain (FDTD) method and the plain wave method (PWM). The FDTD technique allows to simulate the time dependence of a wave packet of vibrational modes propagating through a composite and to determine the transmission coefficient. The PWM method is used for the determination of the phononic dispersion relation in systems with discrete translational symmetry. We use both methods for investigating the effect of periodic interface perturbations on the spectrum of longitudinal vibrational modes in 1D phononic crystals composed of semiconducting materials. The material parameters in the composites under consideration are modulated in the nanoscale.

Key words: phononic crystals, finite difference time domain method, plane wave method
\end{abstract}

\section{INTRODUCTION}

The occurrence of a photonic band gap, or a band of frequencies strictly forbidden to propagating electromagnetic waves in 3D periodic dielectric structures was first suggested by Yablonovitch [1] in 1987. Referred to as photonic crystals, structures with a photonic band gap have by now found many applications in practice. The idea of such macrocrystals was extended to media for other types of classical waves, generally referred to as wave crystals (WC) and including photonic crystals, sonic crystals, elastic wave crystals (a subclass of phononic crystals), plasmonic crystals and magnonic crystals. The common physical property of all these macrocrystals is the occurrence of energy gaps, or bands of disallowed frequencies of propagating waves of the given type. Therefore, most devices using electromagnetic waves and based on photonic crystals have their counterparts that use other types of the classical waves mentioned above, including elastic waves.

The propagation of elastic waves in periodic structures has been the subject of research in recent years [2-8]. Composed of elastic centers (e.g. cylinders, in the two- dimensional case) embedded in a matrix of a different elastic material and disposed in sites of a crystal lattice, phononic crystals show in their band structure frequency ranges forbidden to propagating elastic waves. Due to this property these structures have extensive practical applications, e.g. in the construction of sound shields and filters [9-16], and recently also in refractive devices such as sound-wave focusing acoustic [17] or acoustic interferometers $[17,18]$. Other noteworthy applications of phononic crystals include selective frequency waveguides [19] and acoustic diodes [20].

Phononic crystals with periodicity in a single direction also include superlattices (SL) and multilayers composed of materials of different elastic properties. Vibrational modes in such structures are described by a dispersion relation characteristic of SL, with the folding of phononic branches to the first Brillouin zone of size $\pi / a_{y}$, where $a_{y}$ is the period of the structure. One of the basic properties of SL is the Bragg reflection of elastic waves fulfilling the condition: $2 a_{y} \sin (\alpha)=n \lambda$, where $\alpha$ denotes the angle of incidence measured between the ray of the incident elastic wave and the interface normal, $\lambda$ is the length of the incident wave, and $n$ is an integer. Each phonon that obeys 
this relation is reflected from the structure and cannot propagate through the $\mathrm{SL}$, which is equivalent to the condition of band gap occurrence in $1 \mathrm{D}$ phononic crystals.

In the present study we have performed a numerical investigation of phononic crystals composed of two semiconducting materials, GaAs and AlAs. Such systems have been intensively studied because of their electronic properties: the quantum states and the transport properties. Also their elastic properties arouse interest because of the possibility of reducing the heat transport by appropriate modeling of the phononic dispersion [21]. The conditions at the interfaces between adjacent materials are of major importance for the thermal transport properties [22-25]. Therefore, we investigate the effect of periodic inhomogeneities introduced at interfaces on the phononic spectrum of the SL, in particular on the position and width of the phononic band gaps. Our calculations are based on two complementary methods: the plane wave method (PWM), which allows to determine the dispersion relation for phonons in infinite superlattices, and the finite difference time domain (FDTD) method used for the determination of the transmission coefficient for elastic waves propagating in a crystal of finite thickness.

The paper is organized as follows. In Section II we discuss the FDTD method. Section III presents the PWM technique and the results obtained for an ideal GaAs/AlAs superlattice which can be compared to those reported in the literature. Section IV presents and discusses our results obtained for SL with periodically modulated interfaces. The results are summed up in the closing Section V.

\section{FINITE DIFFERENCE TIME DOMAIN METHOD}

The starting point of our considerations is the wave equation describing the propagation of elastic waves in an inhomogeneous elastic medium [26]:

$$
\frac{\partial^{2} U_{m}}{\partial t^{2}}=\frac{1}{\rho} \frac{\partial T_{m n}}{\partial x_{n}}
$$

where the stress tensor $T_{m n}$ reads:

$$
T_{m n}=\lambda(\vec{r}) U_{l l} \delta_{m n}+2 \mu(\vec{r}) U_{m n},
$$

$l, m$ and $n=1,2$ or 3 , and represents Cartesian coordination's: $x, y$ or $z$, respectively. The deformation tensor $U_{m n}$ has the form:

$$
U_{m n}=\frac{\left(\partial U_{m} / \partial x_{n}+\partial U_{m} / \partial x_{n}\right)}{2},
$$

$\lambda(\vec{r})$ and $\mu(\vec{r})$ are Lamé parameters, and $\rho(\vec{r})$ is the mass density. The Lamé parameters $\lambda$ and $\mu$ can be expressed by the longitudinal and transversal velocity of wave propagation in the material, $c_{l}$ and $c_{t}$, respectively:

$$
\begin{gathered}
\mu=\rho c_{t}^{2}, \\
\lambda=\rho c_{l}^{2}-\rho c_{t}^{2} .
\end{gathered}
$$

For 2D phononic macrocrystals, after the decomposition into the $x$ and $y$ Cartesian components we get a system of two conjugate wave equations [26]:

$$
\begin{gathered}
\frac{\partial^{2} U_{x}}{\partial t^{2}}=\frac{1}{\rho}\left(\frac{\partial T_{x x}}{\partial x}+\frac{\partial T_{x y}}{\partial y}\right), \\
\frac{\partial^{2} U_{y}}{\partial t^{2}}=\frac{1}{\rho}\left(\frac{\partial T_{x y}}{\partial x}+\frac{\partial T_{y y}}{\partial y}\right),
\end{gathered}
$$

where:

$$
\begin{gathered}
T_{x x}=(\lambda+2 \mu) \frac{\partial U_{x}}{\partial x}+\lambda \frac{\partial U_{y}}{\partial y} \\
T_{y y}=(\lambda+2 \mu) \frac{\partial U_{y}}{\partial y}+\lambda \frac{\partial U_{x}}{\partial x} \\
T_{x y}=\mu\left(\frac{\partial U_{x}}{\partial y}+\frac{\partial U_{y}}{\partial x}\right) .
\end{gathered}
$$

Since the $z$ axis is parallel to the cylinder axis, the values of parameters $\lambda(\vec{r}), \mu(\vec{r})$ and $\rho(\vec{r})$ do not depend on the $z$ coordinate. Hence, the solutions of the wave equations along the direction of the translational symmetry, i.e. along the $z$ axis, will be plane waves. As we are only interested in the propagation in the plane of periodicity, we shall look for solutions with wave-vector component $k_{z}=0$. A segment of the composite under consideration, with the

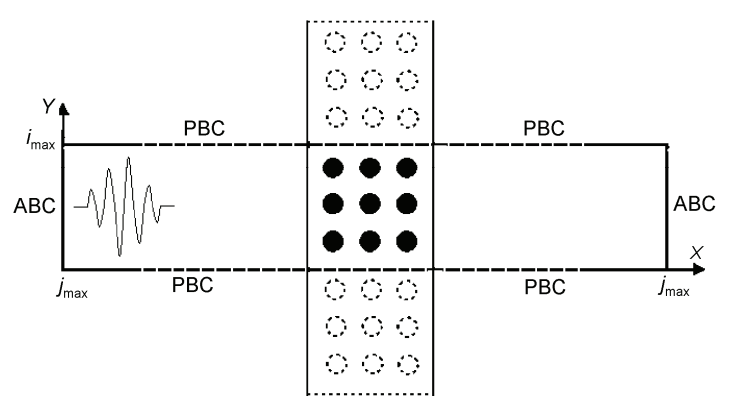

PBC - Periodic Boundary Condition
ABC - Absorption Boundary Condition

Fig. 1. Segment of the composite studied by the FDTD method; periodic boundary conditions (PBC) are set at the $x$ limits (broken lines), and absorption boundary conditions (ABC) at the $y$ limits (solid lines); $y$ is the direction of propagation 
system of coordinates and basic parameters defined, is depicted in Fig. 1.

In the first step of the FDTD method the studied composite is divided to form a discrete lattice of rectangular cells of size $\Delta x \times \Delta y$ along the $x$ and $y$ axes, respectively. This allows to introduce a discrete grid with points indexed $i, j$. The magnitude of the deformation vector component $U_{l}(x, y, t)$ at any point $(i, j)$ of the discretization grid will be defined by the following rule:

$$
U_{l}(i, j, k)=U_{l}\left(i \Delta x-\frac{1}{2} \Delta x ; j \Delta y-\frac{1}{2} \Delta y, k \Delta t\right),
$$

where $l=x, y$ only; $i \geq 1, j \geq 1$ and $k \geq 0$.

The additional index $k$ is the calculation time count with step $\Delta t$. The time step must be appropriately adjusted to assure the convergence of the results obtained [26-28]; we shall discuss this issue further in this Section.

In the next step the wave equation is discretized by the approximation of the space and time derivatives in Equations (5) and (6) to finite differences at points $(i, j, k)$. Central differences are used in the case of space derivatives:

$$
\begin{gathered}
\left.\frac{\partial U_{l}}{\partial x}\right|_{i, j, k} \approx D_{0}^{x} U_{l}(i, j, k)= \\
=\frac{\left[U_{l}\left(i+\frac{1}{2}, j, k\right)-U_{l}\left(i-\frac{1}{2}, j, k\right)\right]}{\Delta x}, \\
\left.\frac{\partial U_{l}}{\partial y}\right|_{i, j, k} \approx D_{0}^{y} U_{l}(i, j, k)= \\
=\frac{\left[U_{l}\left(i, j+\frac{1}{2}, k\right)-U_{l}\left(i, j-\frac{1}{2}, k\right)\right]}{\Delta y} .
\end{gathered}
$$

The second time derivative of the deformation vector in Equations (5) and (6) is represented as the product of two difference operators, $D_{+}^{t}$ and $D_{-}^{t}[26]$ :

$$
\left.\frac{\partial^{2} U_{l}}{\partial t^{2}}\right|_{i, j, k} \approx D_{+}^{t} D_{-}^{t} U_{l}(i, j, k),
$$

where

$$
\begin{aligned}
& D_{+}^{t} U_{l}(i, j, k)=\frac{U_{l}(i, j, k+1)-U_{l}(i, j, k)}{\Delta t}, \\
& D_{-}^{t} U_{l}(i, j, k)=\frac{U_{l}(i, j, k)-U_{l}(i, j, k-1)}{\Delta t},
\end{aligned}
$$

and still $l=x$ or $y$.

Replacing the derivatives in (5) with the above finite differences leads to the following difference equation:

$$
\begin{aligned}
& U_{x}(i, j, k+1)=2 U_{x}(i, j, k)-U_{x}(i, j, k-1)+ \\
& +\frac{\Delta_{t}^{2}}{\rho(i, j) \Delta_{x}}\left[T_{x x}\left(i+\frac{1}{2}, j, k\right)-T_{x x}\left(i-\frac{1}{2}, j, k\right)\right]+ \\
& +\frac{\Delta_{t}^{2}}{\rho(i, j) \Delta_{y}}\left[T_{x y}\left(i, j+\frac{1}{2}, k\right)-T_{x y}\left(i, j-\frac{1}{2}, k\right)\right],
\end{aligned}
$$

where the formulas for $T_{x x}, T_{y y}$ and $T_{x y}$ are as specified in Appendix A at the end of this paper, and $\rho(i, j)$ is the material density at point $(i \Delta x, j \Delta y)$.

By a similar procedure applied to the derivatives in (6) we get the formula for $U_{y}\left(i+\frac{1}{2}, j+\frac{1}{2}, k+1\right)$ :

$$
\begin{gathered}
U_{y}\left(i+\frac{1}{2}, j+\frac{1}{2}, k+1\right)= \\
=2 U_{y}\left(i+\frac{1}{2}, j+\frac{1}{2}, k\right)-U_{y}\left(i+\frac{1}{2}, j+\frac{1}{2}, k-1\right)+ \\
+\frac{\Delta_{t}^{2}}{\rho\left(i+\frac{1}{2}, j+\frac{1}{2}\right) \Delta_{x}}\left[T_{x y}\left(i+1, j+\frac{1}{2}, k\right)-T_{x y}\left(i, j+\frac{1}{2}, k\right)\right]+ \\
+\frac{\Delta_{t}^{2}}{\rho\left(i+\frac{1}{2}, j+\frac{1}{2}\right) \Delta_{y}}\left[T_{y y}\left(i+\frac{1}{2}, j+1, k\right)-T_{y y}\left(i+\frac{1}{2}, j, k\right)\right] .
\end{gathered}
$$

Below we present the algorithm of the FDTD-based determination of the transmission coefficient for elastic waves propagating in a $2 \mathrm{D}$ (or a 1D) phononic crystal:

1. Establish the geometry and size of the composite by the appropriate settings of the material and structural parameters.

2. Use a rectangular grid to divide the composite into small $\Delta x \times \Delta y$ cells. The values of $\Delta x$ and $\Delta y$ are determined by the established density of the grid.

3. Set the time step $\Delta t$, which must meet the stability condition:

$$
\Delta t \leq \frac{1}{2 c \sqrt{\frac{1}{\Delta x^{2}}+\frac{1}{\Delta y^{2}}}},
$$

where $c$ is the highest of the velocity values, longitudinal or transversal, in one of the component materials of the composite under consideration. The step $\Delta t$ must be adjusted so as to prevent an unlimited increase of results of the numerical calculations with time, i.e. assure their convergence.

4. Set the initial conditions or generate a wave packet at one side of the composite (left in this paper) starting from time $t=0$ till the entire packet is formed. For this purpose, the magnitudes of the components of the 
deformation vector $U_{l}$ are calculated on the grid $(i, j)$ by formulas:

$$
\begin{aligned}
& U_{y}=\alpha \sin \left(\omega t-\frac{y}{c_{l}}\right) \exp \left[-\beta\left(\omega t-\frac{y}{c_{l}}\right)^{2}\right], \\
& U_{x}=\alpha \sin \left(\omega t-\frac{y}{c_{t}}\right) \exp \left[-\beta\left(\omega t-\frac{y}{c_{t}}\right)^{2}\right],
\end{aligned}
$$

where the factor $\alpha$ is the maximum wave amplitude (for convenience we assume its value to be comparable with the size of the composite along the $x$ axis); $c_{l} / \beta$ is the full width at half maximum.

5. Perform the simulation. At each successive moment $(k>1)$ and each point $(i, j)$ of the grid, with the exception of the limiting points $y=j_{\min }$ and $y=j_{\max }$, calculate the values of $U_{l}$ using the formulas (14) and (15). The values of potential $U_{l}$ at intermediate points not included in the grid should be calculated as the arithmetic mean of $U_{l}$ values at neighboring grid points.

6. Set the boundary conditions. It should be emphasized that the system studied by the finite difference method must always be finite. This implies the necessity of setting adequate boundary conditions at the limits of the calculation area; from a number of possibilities, in our calculations we assume periodic boundary conditions along the $y$ axis and absorption boundary conditions along the $x$ axis. The periodic boundary conditions along the $x$ axis read:

$$
\begin{gathered}
U_{l}\left(i_{\max }+1, j, k\right)=U_{l}(1, j, k), \\
U_{l}\left(i_{\max }, j, k\right)=U_{l}(0, j, k),
\end{gathered}
$$

where $i=i_{\max }$ and $i=0$ correspond to the last and the first plane of the calculation area, respectively; $l=x$ or $y$. Along the direction of propagation (the $y$ direction), i.e. for points with indices $j=j_{\min }$ and $j=j_{\max }$, we set Mur's first-order absorption boundary conditions [29]. Thus:

$$
\begin{aligned}
& U_{l}(i, 0, k+1)=U_{l}(i, 1, k)+ \\
& +\frac{c \Delta t-\delta}{c \Delta t+\delta} \cdot\left[U_{l}(i, 1, k+1)-U_{l}(i, 0, k)\right] \\
& \text { for } j=0, \\
& \quad U_{l}\left(i, j_{\max }, k+1\right)=U_{l}\left(i, j_{\max }-1, k\right)+ \\
& +\frac{c \Delta t-\delta}{c \Delta t+\delta} \cdot\left[U_{l}\left(i, j_{\max }-1, k+1\right)-U_{l}\left(i, j_{\max }, k\right)\right] \\
& \text { for } j_{\max },
\end{aligned}
$$

where $\delta=\Delta x$ for $l=x, \delta=\Delta y$ for $l=y$, and $c$ is the longitudinal or transversal velocity of wave propaga- tion, respectively, in the material at the extremities of the composite under consideration.

7. Perform the Fourier transformation of the obtained time dependence $U_{l}(t)$ to the frequency space $U_{l}(\omega)$ by the fast Fourier transformation technique.

8. Determine the transmission coefficient $\Gamma$ as the amplitude ratio of the outgoing wave (out) (at the right side of the composite) to the incoming signal (in), by the formula:

$$
\Gamma=\frac{\left.\sqrt{U_{x}^{2}(\omega)+U_{y}^{2}(\omega)}\right|_{\text {out }}}{\left.\sqrt{U_{x}^{2}(\omega)+U_{y}^{2}(\omega)}\right|_{\text {in }}} .
$$

We tested the method and the developed computer program by carrying out numerical simulations of longitudinal modes of elastic waves propagating through a superlattice composed of two semiconductors, GaAs and AlAs. Such systems have already been studied intensively and reference data are available in the literature. Considering the subject matter of this study, we decided to refer to a paper by S. Mizuno et al. [30] and use the parameter values specified therein. Thus, we performed a full simulation for a multilayer structure composed of two subsystems. Subsystem 1 is a multilayer consisting of 20 layers of AlAs, each 6 monolayer (ML) thick, alternating with GaAs layers of the same thickness. Subsystem 2 is a composite consisting of 15 layers, each $9 \mathrm{ML}$ thick, alternating with GaAs layers of the same thickness. The assumed thickness of a single AlAs or GaAs monolayer along the [100] direction is $2.83 \AA$, which means the total thickness of the system is $510 \AA$.

The results of the numerical simulations are shown in Fig. 2 (dotted line). In the same Figure we indicate the values of transmission coefficient obtained by the transfer matrix method in paper [30]. Two band gaps, or frequency ranges with a very low transmission coefficient, are seen to occur in the frequency range from $0.45 \mathrm{THz}$ to $0.85 \mathrm{THz}$. The occurrence of two gaps is a consequence of the presence of two subsystems with respective lattice constant values $12 \mathrm{ML}$ and $18 \mathrm{ML}$. The lower-frequency gap corresponds to the latter. It should be stressed that though the results are in qualitative agreement with the literature data, quantitative differences persist and could not be eliminated by increasing the accuracy of the discretization step. The main difference is a shift of the bottom limits of both band gaps to higher frequency in the simulation results. This is probably due to the way of determination of the transmission coefficient. Mizuno et al. [30] determine $\Gamma$ from equations that only describe the propagation of longitudinal waves, while our FDTD simulations are based on full conjugate equations describing both the longitudinal and the transversal waves, hence the observed transmission differences. 


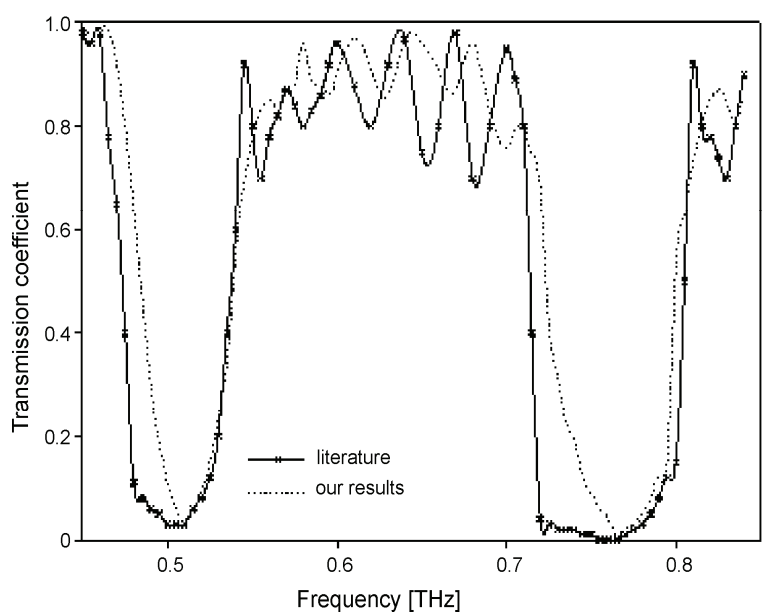

Fig. 2. The transmission coefficient vs. frequency for elastic waves propagating through $1 \mathrm{D}$ phononic crystal consisting of two composites. Composite 1 comprises 20 layers of AlAs, each $6 \mathrm{ML}$ thick, alternating with GaAs layers of the same thickness. Composite 2 consists of 15 layers, each 9 ML thick, alternating with GaAs layers of the same thickness. Solid and dotted lines represent data from [30] and the results of our numerical simulations, respectively

\section{PLANE WAVE METHOD}

In this Section we present the technique of calculating the phononic band structure based on solving the elastic medium wave Equation (1) by the well-known plane wave method (PWM) [5, 31, 32]. In this technique, the differential wave Equation (1), in which the coefficients are periodic functions of two-dimensional position vector $\vec{r}=(x, y)$, is transformed into the reciprocal space.

As the structure considered in this paper is homogeneous along the $z$ axis, the problem reduces to two dimensions. Moreover, if the wave propagation is confined to the plane of periodicity of the phononic crystal, Eq. (1) splits into two independent equations [32]:

i) an equation describing XY modes, or oscillations polarized in plane $(x, y)$ :

$$
\frac{\partial^{2} U_{m}}{\partial t^{2}}=\frac{1}{\rho}\left\{\frac{\partial}{\partial x_{m}}\left(\lambda \frac{\partial U_{l}}{\partial x_{l}}\right)+\frac{\partial}{\partial x_{l}}\left[\mu\left(\frac{\partial U_{m}}{\partial x_{l}}+\frac{\partial U_{l}}{\partial x_{m}}\right)\right]\right\},
$$

where $m, l=1,2$ (Cartesian $x$ or $y$ components, respectively), and ii) an equation describing $Z$ modes, or oscillations polarized along the rod axis, i.e. along the $z$ axis:

$$
\frac{\partial^{2} U_{z}}{\partial t^{2}}=\frac{1}{\rho} \nabla \cdot\left[\mu \nabla U_{z}\right]
$$

As the coefficients in the equations of motion (16) and (17) are periodic functions, by virtue of Bloch's theorem the deformation vector $\vec{U}(\vec{r}, t)$ can be expressed as [31]:

$$
\vec{U}(\vec{r}, t)=e^{i(\vec{k} \cdot \vec{r}-\omega t)} \sum_{\vec{G}} \vec{U}_{\vec{k}}(\vec{G}) e^{i \vec{G} \cdot \vec{r}}
$$

$\vec{k}$ being a two-dimensional Bloch vector, and $\vec{G}$ denoting a two-dimensional reciprocal lattice vector.

Those of the material parameters which are periodic functions can be Fourier-expanded. For $Z$ modes, described by (17), Fourier expansion can be applied to the inverse of mass density, $\rho^{-1}$, and to the coefficient $\mu$; in the case of $\mathrm{XY}$ modes also the coefficient $\lambda$ can be Fourier-expanded:

$$
\begin{gathered}
\rho^{-1}(\vec{r})=\sum_{\vec{G}} \rho^{-1}(\vec{G}) e^{i \vec{G} \cdot \vec{r}}, \quad \mu(\vec{r})=\sum_{\vec{G}} \mu(\vec{G}) e^{i \vec{G} \cdot \vec{r}}, \\
\lambda(\vec{r})=\sum_{\vec{G}} \lambda(\vec{G}) e^{i \vec{G} \cdot \vec{r}} .
\end{gathered}
$$

All the coefficients, $\rho^{-1}(\vec{G}), \mu(\vec{G}), \lambda(\vec{G})$, in the above expansions are calculated from the inverse Fourier transform and will have the same form; for example, the inverse of mass density will read:

$$
\rho^{-1}(\vec{G})=\frac{1}{S} \iint \rho^{-1}(\vec{r}) e^{-i \vec{G} \cdot \vec{r}} d \vec{r},
$$

where the integration is performed over the surface $S$ of the $2 \mathrm{D}$ unit cell. These integrations can be done analytically only for regular shapes, and numerically otherwise. For superlattices of the type considered in this paper, with periodically corrugated interfaces, the integration can be performed analytically; the respective formulas are presented in Appendix B.

The substitution of (18) and (19) into (16) leads to an infinite system of algebraic equations for XY mode eigenvalues $\omega^{2}(\vec{k})$ :

$$
\omega^{2} U_{m, \vec{k}}(\vec{G})=\sum_{\vec{G}^{\prime}}\left\{\begin{array}{l}
\sum_{l, \vec{G}^{\prime \prime}} \rho^{-1}\left(\vec{G}-\vec{G}^{\prime \prime}\right)\left[\begin{array}{l}
\lambda\left(\vec{G}^{\prime \prime}-\vec{G}^{\prime}\right)\left(\vec{k}+\vec{G}^{\prime \prime}\right)_{l}\left(\vec{k}+\vec{G}^{\prime \prime}\right)_{m} \\
+\mu\left(\vec{G}^{\prime \prime}-\vec{G}^{\prime}\right)\left(\vec{k}+\vec{G}^{\prime}\right)_{m}\left(\vec{k}+\vec{G}^{\prime \prime}\right)_{l}
\end{array} U_{l, \vec{k}}\left(\vec{G}^{\prime}\right)\right. \\
+\sum_{\vec{G}^{\prime \prime}}\left[\rho^{-1}\left(\vec{G}-\vec{G}^{\prime \prime}\right) \mu\left(\vec{G}^{\prime \prime}-\vec{G}^{\prime}\right) \sum_{n}\left(\vec{k}+\vec{G}^{\prime}\right)_{n}\left(\vec{k}+\vec{G}^{\prime \prime}\right)_{n}\right]_{m, \vec{k}^{\prime}}\left(\vec{G}^{\prime}\right)
\end{array}\right\},
$$


where $m, n, l=1,2$ ( $x$ or $y$ respectively). Analogically, the following $Z$ mode eigenvalue problem is deduced from (17):

$$
\begin{gathered}
\omega^{2} U_{z, \vec{k}}(\vec{G})= \\
=\sum_{\vec{G}^{\prime}, \vec{G}^{\prime \prime}} \rho^{-1}\left(\vec{G}-\vec{G}^{\prime \prime}\right) \mu\left(\vec{G}^{\prime \prime}-\vec{G}^{\prime}\right)\left(\vec{k}+\vec{G}^{\prime}\right) \cdot\left(\vec{k}+\vec{G}^{\prime \prime}\right) U_{z, \vec{k}}\left(\vec{G}^{\prime}\right)
\end{gathered}
$$

In the numerical solution of (21) and (22) we used standard functions from the GSL library [33] for solving the eigenvalue problem, i.e. gsl_eigen_nonsymm. The number of reciprocal lattice vectors involved in Fourier expansions (18)-(19) is limited by the condition $-N \leq n_{x}, n_{y} \leq N$, confining the magnitudes $n_{x}$ and $n_{y}$ of the reciprocal lattice vector components to the interval $\langle-N, N\rangle$. From the resulting finite system of algebraic equations $6 N+3$ allowed frequencies $\omega(\vec{k})$ are deduced for each wave vector from the first Brillouin zone. All the results presented in this paper were obtained for $N=7$.

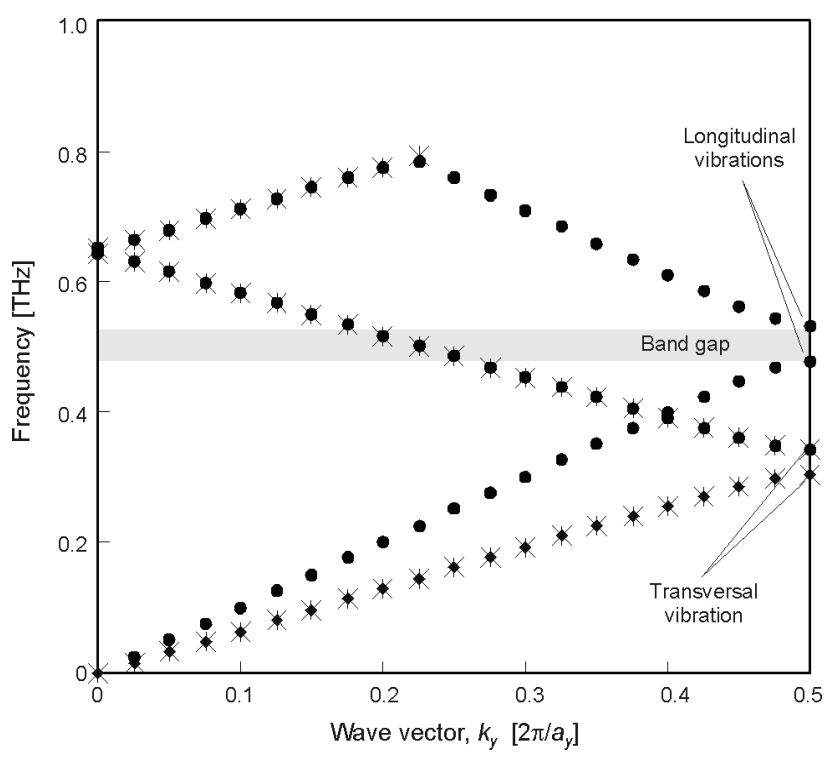

Fig. 3. Phononic band structure calculated by the plane wave method for superlattice consisting of $25.47 \AA$ (9 ML) thick GaAs layers alternating with AlAs layers of the same thickness. Stars and dark dots represent branches of $\mathrm{Z}$ modes obtained from (22) and branches resulting from the solution of (21), respectively. Longitudinal modes correspond to the elastic waves studied in [30], the phononic band gap for longitudinal modes is marked in gray. For verification purposes the material parameter values used in the calculations are as specified in [30]

First let us apply the PWM to a simple system represented by a superlattice consisting of alternating GaAs and AlAs layers, and thus periodic only in one direction, namely the $y$ direction, which is also the propagation direction of the elastic wave. This will allow us to test our method by comparing the results obtained to those reported in the literature [30] and also to those obtained by the FTDT method in the previous Section, and, at the same time, to explain the basic properties of the spectrum of elastic waves propagating in phononic crystals. Figure 3 presents the elastic wave frequencies versus the first Brillouin zone wave vector component $k_{y}$. Three types of branches are seen in the plot: two resulting from the numerical solution of (21) (dark dots), and one, corresponding to $\mathrm{Z}$ modes, obtained from (22) (stars).

As the system is undistinguishable along the directions perpendicular to the direction of periodicity, modes polarized along the $z$ axis ( $Z$ modes) and those polarized along the $x$ axis must have the same frequencies. This is confirmed by the results depicted in Fig. 3, showing double-degenerate branches corresponding to transversal vibrations and branches of longitudinal modes vibrating along the propagation direction. The propagation of longitudinal modes in such systems has already been studied in the preceding Section and by Mizuno et al. [30], by the FDTD method and the transfer matrix method, respectively. In the PWM technique the superlattice is regarded as an equivalent of subsystem 2 of the composite discussed in Section II. The comparison of results indicates a very good agreement in terms of the position and width of the first band gap in the spectrum of longitudinal modes. This allows us to proceed to further investigations.

\section{PHONONIC SUPERLATTICES WITH PERIODICALLY CORRUGATED INTERFACES}

Let us investigate the effect of a periodic modulation of interfaces on the spectrum of vibrations of semiconductor superlattices. We shall start from PWM calculations. The unit cell of the superstructure considered is as defined in Fig. 4. By a periodic repetition of the unit cell we get a superstructure based on a rectangular lattice with lattice vectors $\left(a_{x}, 0\right)$ and $\left(0, a_{y}\right)$; the interface perturbations are modeled by cylinders of radius $r$ disposed periodically along the $x$ axis and parallel to the $z$ axis. The assumed layer thickness values are $9 \mathrm{ML}$ and $18 \mathrm{ML}$ for GaAs (material A) and for material B (AlAs), respectively. In the case of unperturbed interfaces (cylinder radius $r=0.0$ ) we get a simple superlattice with the dispersion relation as shown in Fig. 5, by empty circles (all the material parameters used in the calculations are specified in Table 1). We can see two phononic band gaps between: $0.33-0.36 \mathrm{THz}$ and $0.68-0.71 \mathrm{THz}$. The introduction of cylinders with radius $r=5.094 \AA$, spacing $a_{x}=22.923 \AA$ 
made from the same material as A (i.e. GaAs), results in a slightly modified dispersion relation (Fig. 5(a), crosses). The frequencies of almost all the modes are shifted down; the shift clearly increases with frequency and is greatest at the limits and in the center of the Brillouin zone but only for the bands just under the gaps (as seen in the enlarged segments of the plot, Fig. 5(b) and (c)). This behavior can be explained by the localization of modes connected with upper branches in perturbed regions. Also depicted in Fig. 5 is the dispersion relation for longitudinal phonons

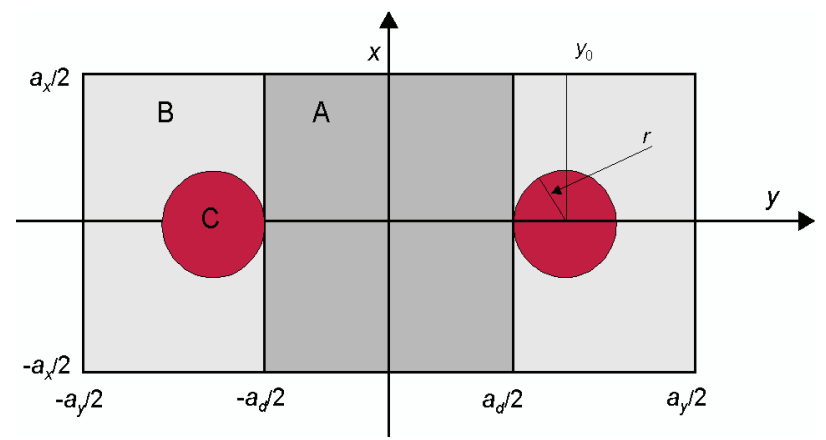

Fig. 4. Unit cell of phononic superlattice with periodically modulated interfaces. The interface modulation is modeled by cylinders of material $\mathrm{C}$ and radius $r$ attached to material A (with thickness $a_{d}$ ); thus, the $y$ distance between the origin of the coordinate system and the center of each cylinder is $y_{0}=1 / 2 a_{d}+r$

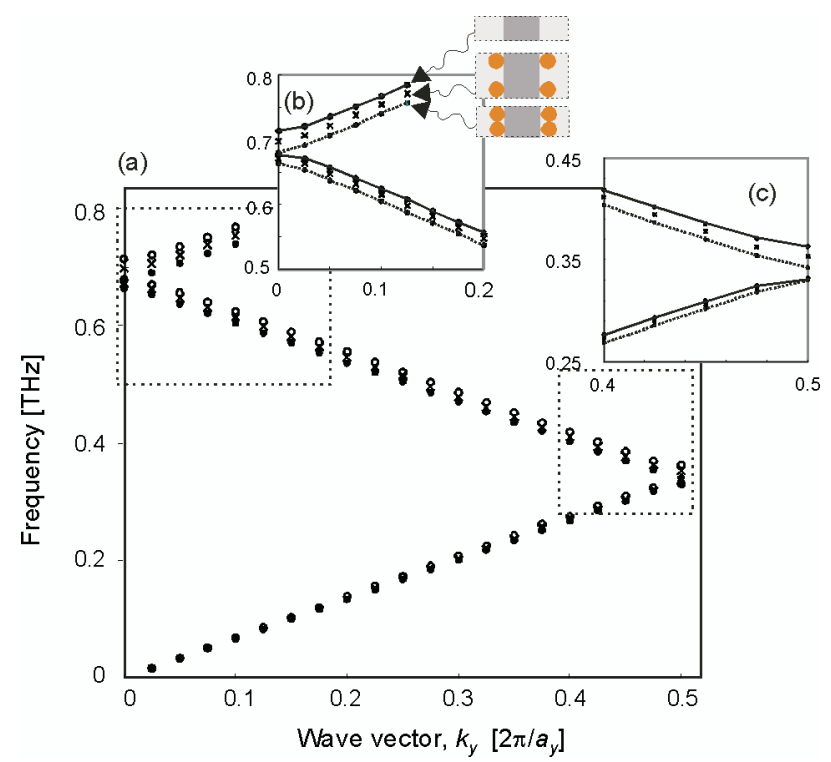

Fig. 5. (a) Dispersion of longitudinal vibrations propagating along the $y$ axis in superlattice consisting of 9 ML thick GaAs layers alternating with $18 \mathrm{ML}$ thick AlAs layers: empty circles, crosses and full circles correspond to the cases with unperturbed interfaces, with cylinders distributed sparsely along the interfaces

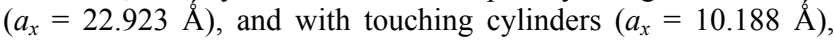
respectively; (b) and (c) present enlarged segments of the dispersion plot (a) around the first and the second band gap, respectively
Table 1. Material parameters of GaAs and AlAs used for the determination of the transmission coefficient and in the PWM calculations for 1D phononic crystal with periodically perturbed interfaces

\begin{tabular}{c|c|c|c|c|c}
\cline { 2 - 6 } & $\begin{array}{c}c_{l} \\
{[\mathrm{~m} / \mathrm{s}]}\end{array}$ & $\begin{array}{c}c_{t} \\
{[\mathrm{~m} / \mathrm{s}]}\end{array}$ & $\begin{array}{c}\rho \\
{\left[\mathrm{kg} / \mathrm{m}^{3}\right]}\end{array}$ & $\begin{array}{c}\lambda \\
{\left[10^{3}\right]}\end{array}$ & $\begin{array}{c}\mu \\
{\left[10^{3}\right]}\end{array}$ \\
\hline $\mathrm{AlAs}$ & 5980 & 3600 & 3760 & 36999904 & 48729600 \\
\hline $\mathrm{GaAs}$ & 5030 & 3030 & 5320 & 36916012 & 48842388 \\
\hline
\end{tabular}

with perturbation spacing $a_{x}=10.188 \AA$, which corresponds to adjacent cylinders touching one another; the corresponding dispersion is indicated by full circles. The previously observed changes are seen to continue.

Let us now apply the FDTD method presented and tested in Section II to the determination of the transmission coefficient for elastic waves passing through a multilayer system with interface perturbations as described above. Table 1 presents the material parameters used in a number

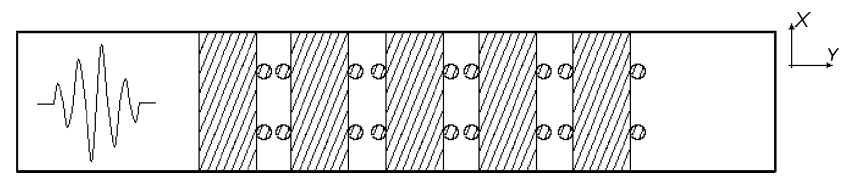

Fig. 6. One-dimensional phononic crystal with periodically perturbed interfaces. The system depicted corresponds to the composite assumed in the FDTD simulations. Layers of AlAs, each composed of 9 AlAs monolayers (white areas), alternate with $18 \mathrm{ML}$ thick layers of GaAs (shadow areas). Interface perturbations are modeled by the addition of cylinders (parallel to the $z$ axis) with radius $r$ and spacing $a_{x}$, made of GaAs. The unit cell of the system with discrete translational symmetry used in PWM calculations, corresponding to this composite, is shown in Fig. 4
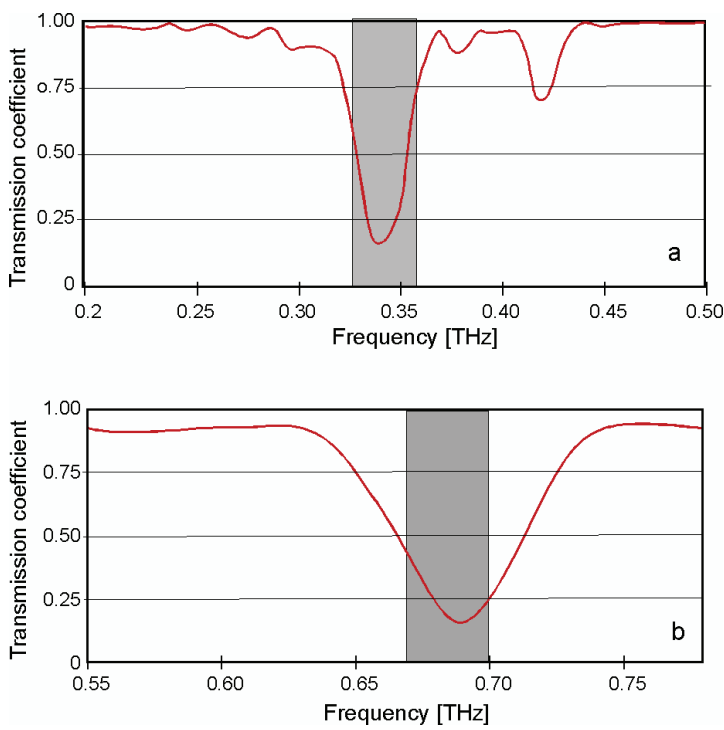

Fig. 7. Transmission coefficient versus frequency in the 1D phononic crystal schematically depicted in Fig. 6, with periodically perturbed interfaces. As the gray area we have marked the phononic band gap received from Plane Wave Method 
of simulations performed for wave packets generated on the left side of the composite at different frequencies in the range from $0.2 \mathrm{THz}$ to $0.5 \mathrm{THz}$ and from $0.55 \mathrm{THz}$ to $0.8 \mathrm{THz}$, i.e. around both band gaps found with the PWM. The phononic crystal studied in the FDTD simulations is schematically depicted in Fig. 6.

Figure 7 shows the frequency dependence of the transmission coefficient in the structure defined above, calculated by the FDTD method. The following parameter values were assumed in the calculations: radius $r=5.094 \AA$ of the cylindrical perturbation, perturbation spacing $a_{x}=31.32 \AA$ along the $x$ axis, height $x=93.96 \AA$ of the omposite (along the $x$ direction), total width $y=$ $3.0564 \mu \mathrm{m}$ of the composite (along the propagation direction). The discretization step is $\Delta x=1.3423 \AA$ and $\Delta y$ $=1.698 \AA$ in the $x$ and $y$ direction, respectively. A first phononic gap is seen to occur around the frequency of $0.35 \mathrm{THz}$. Also deep around the frequency of $0.69 \mathrm{THz}$ in transmission spectra, despite its very blurred edges, represents an actual phononic gap, as confirmed by the images of a propagating wave packet at time step 30000 from the beginning of the simulation presented in Fig. 8. Figure 8(a) shows such a wave packet ( $U_{y}$ component of the deformation vector) of frequency $0.690 \mathrm{THz}$, the central frequency of the gap; a majority of the wave packet is reflected by the composite and only a minor part reaches the detector at the opposite side. The situation is reversed in the case of the $0.770 \mathrm{THz}$ wave depicted in Fig. 8(b). Almost the entire packet passes through the composite, and only a small part is reflected, which is a clear evidence that this wave frequency is already beyond the gap.
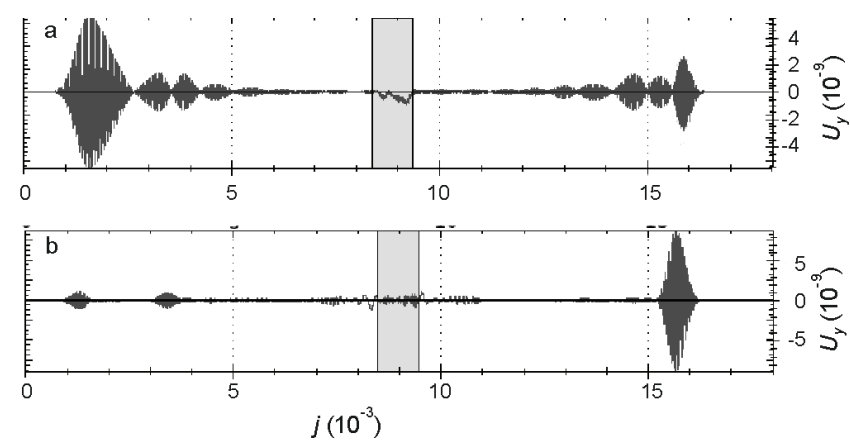

Fig. 8. $U_{y}$ component of the deformation vector of elastic wave propagating through phononic crystal with perturbed interfaces, as obtained in FDTD simulations at time step $k=30$ 000: a) a wave packet of frequency $0.690 \mathrm{THz}$, within the energy gap (Fig. 7); b) a wave packet of frequency $0.770 \mathrm{THz}$, beyond the energy gap. The composite is situated in the middle and marked by the gray area

The obtained band gaps agree very well with those from PWM, as one can see in Fig. 7, where by gray areas the phononic band gaps calculated with PWM are marked, too. They have sharp borders, as usually is for infinite crystals and opposite to finite ones. But further investigations are necessary to explain in details quite big broadening of the second phononic gap obtained in FTDT simulations. This effect could also be attributed to conjugation of longitudinal and transversal modes.

\section{SUMMARY}

We have used two techniques, the plane wave method and the finite difference time domain method, for calculating the dispersion relation and the transmission coefficient for elastic waves propagating in phononic crystals. Of these two complementary numerical techniques, the PWM method has a number of limitations, including the requirement of an infinite size of the studied composite, the components of which, moreover, must be in the same phase. These limitations do not apply in the FDTD method; therefore, this technique can be used for simulating the propagation of waves through finite composites, which are better models of the experimental systems. However, also the FDTD method has major disadvantages, such as the necessity of using a dense discretization grid, which results in very long calculations compared to those based on the PWM technique. Moreover, a single FDTD simulation provides information on the system response to the incident wave of only one frequency, while in the PWM technique the full dispersion relation is obtained.

We have verified and then used both methods for studying vibrational modes and the transmission of elastic waves through GaAs/AlAs semiconductor superlattices. We focused on the effect of a periodic modulation introduced at the interfaces between GaAs and AlAs on the position and width of the phononic band gaps. The interface modulation was modeled by cylindrical rods of GaAs disposed along the interfaces. This extra perturbation introduced to the phononic crystal is found to cause only minor modifications of the band gaps of longitudinal modes: the shift down of the phononic bands. The most significant shift is observed for modes laying just under the gaps, this can be attributed to localization of those vibrations in perturbed regions. Therefore, the periodic interface corrugations can be expected to have a minor effect on the phononic gaps also in real systems, but can allow for independent modeling of phononic band gap edges in some range of frequency. Further detailed calculations are necessary to precisely describe the role of different kind periodic interface perturbations on phononic band gaps and these studies are under investigation. 


\section{Acknowledgements}

The calculations presented in this paper were performed in Poznań Supercomputing and Networking Center.

\section{Appendix A}

In this Appendix we present the formulas for coefficients $T_{x x}, T_{y y}, T_{x y}$ in Equations (14) and (15) used in numerical simulations based on the FDTD method:

$$
\begin{gathered}
T_{x x}\left(i+\frac{1}{2}, j, k\right)= \\
=\frac{(\lambda+2 \mu)_{\left(i+\frac{1}{2}, j\right)}\left[U_{x}(i+1, j, k)-U_{x}(i, j, k)\right]}{\Delta x}+ \\
+\frac{\lambda_{\left(i+\frac{1}{2}, j\right)}\left[U_{y}\left(i+\frac{1}{2}, j+\frac{1}{2}, k\right)-U_{y}\left(i+\frac{1}{2}, j-\frac{1}{2}, k\right)\right]}{\Delta y}, \\
+\frac{(\lambda+2 \mu)_{\left(i-\frac{1}{2}, j\right)}\left[U_{x}(i, j, k)-U_{x}(i-1, j, k)\right]}{\Delta x}+ \\
\mu_{\left(i, j-\frac{1}{2}\right)}\left[U_{y}\left(i+\frac{1}{2}, j-\frac{1}{2}, k\right)-U_{y}\left(i-\frac{1}{2}, j-\frac{1}{2}, k\right)\right] \\
\Delta x
\end{gathered}
$$

$$
\begin{gathered}
T_{x y}\left(i+1, j+\frac{1}{2}, k\right)= \\
=\frac{\mu_{\left(i+1, j+\frac{1}{2}\right)}\left[U_{x}(i+1, j+1, k)-U_{x}(i+1, j, k)\right]}{\Delta y}+ \\
+\frac{\mu_{\left(i+1, j+\frac{1}{2}\right)}\left[U_{y}\left(i+\frac{3}{2}, j+\frac{1}{2}, k\right)-U_{y}\left(i+\frac{1}{2}, j+\frac{1}{2}, k\right)\right]}{\Delta x},
\end{gathered}
$$

$$
\begin{gathered}
T_{y y}\left(i+\frac{1}{2}, j+1, k\right)= \\
=\frac{(\lambda+2 \mu)_{\left(i+\frac{1}{2}, j+1\right)}\left[U_{y}\left(i+\frac{1}{2}, j+\frac{3}{2}, k\right)-U_{y}\left(i+\frac{1}{2}, j+\frac{1}{2}, k\right)\right]}{\Delta y}+ \\
+\frac{\lambda_{\left(i+\frac{1}{2}, j+1\right)}\left[U_{x}(i+1, j+1, k)-U_{x}(i, j+1, k)\right]}{\Delta x}, \\
=\frac{(\lambda+2 \mu)_{\left(i+\frac{1}{2}, j\right)}\left[U_{y}\left(i+\frac{1}{2}, j+\frac{1}{2}, k\right)-U_{y}\left(i+\frac{1}{2}, j-\frac{1}{2}, k\right)\right]}{\Delta y}+ \\
+\frac{\lambda_{\left(i+\frac{1}{2}, j\right)}\left[U_{x}(i+1, j, k)-U_{x}(i, j, k)\right]}{\Delta x} .
\end{gathered}
$$

\section{Appendix B}

Below we present the Fourier coefficients of periodic material parameters used in (21) and (22) for a phononic SL with periodic interface modulations as described above in this paper, and with unit cell as depicted in Fig. 4. Thus, the SL consists of two materials, $\mathrm{A}$ and $\mathrm{B}$, of respective thickness $a_{d}$ and $a_{y}$, and the periodic modulations of their interfaces are modeled by small cylinders of material $\mathrm{C}$ with radius $r$. The $y$ and $x$ dimensions of the unit cell are $a_{y}$ and $a_{x}$, respectively. The Fourier coefficients of the periodic material parameters can be calculated from the following equations:

$$
F(\vec{G})= \begin{cases}F_{A} a_{d} a_{x}+2 F_{C} \pi r^{2}+F_{B}\left(a_{x} a_{y}-a_{d} a_{x}-2 \pi r^{2}\right) & \text { when } \vec{G}=0, \\ \frac{1}{a_{x} a_{y}}\left[\left(F_{A}-F_{B}\right) a_{x} a_{d} \frac{\sin \left(\frac{G_{x} a_{d}}{2}\right)}{\left.\frac{G_{x} a_{d}}{2}+4\left(F_{C}-F_{B}\right) \pi \rho \cos \left(G_{x} y_{0}\right) \frac{J_{1}\left(G_{x} r\right)}{G_{x}}\right]}\right. & \text { when } G_{x} \neq 0 \text { and } G_{y}=0, \\ \frac{4}{a_{x} a_{y}}\left(F_{C}-F_{B}\right) \pi r \cos \left(G_{x} y_{0}\right) \frac{J_{1}\left(G_{y} r\right)}{G_{y}} & \text { when } G_{x}=0 \text { and } G_{y} \neq 0, \\ \frac{4}{a_{x} a_{y}}\left(F_{C}-F_{B}\right) \pi r \cos \left(G_{x} y_{0}\right) \frac{J_{1}(G r)}{G} & \text { when } G_{x} \neq 0 \text { and } G_{y} \neq 0,\end{cases}
$$


where $F(\vec{G})$ stands for $\rho^{-1}(\vec{G}), \mu(\vec{G})$ or $\lambda(\vec{G})$, and $F_{A}, F_{C}$, $F_{B}$ for the respective material parameters in material A, B or $\mathrm{C}$; $J_{l}$ is a Bessel function of the first kind, $G$ is the length of the reciprocal lattice vector, and $G_{x}, G_{y}$ are its Cartesian components.

\section{References}

[1] E. Yablonovitch, Inhibited spontaneous emission in solidstate physics and electronics. Phys. Rev. Lett. 58, 20592062 (1987).

[2] E.N. Economou, M. Sigalas, Stop bands for elastic-waves in periodic composite-materials. J. Acoust. Soc. Am. 95, 1734-1740 (1994).

[3] M.S. Kushwaha, P. Halevi, Band-gap engineering in periodic elastic composites. Appl. Phys. Lett. 64, 1085-1087 (1994).

[4] M.S. Kushwaha, P. Halevi, L. Dobrzynski, B. DjafariRouhani, Acoustic band structure of periodic elastic composites. Phys. Rev. Lett. 71, 2022-2025 (1993).

[5] M.S. Kushwaha, P. Halevi, G. Martinez, L. Dobrzynski, B. Djafari-Rouhani, Theory of acoustic band structure of periodic elastic composites. Phys. Rev. B 49, 2313-2322 (1994).

[6] M. Sigalas, E.N. Economou, Elastic waves in plates with periodically placed inclusions. J. Appl. Phys. 75, 28452850 (1994).

[7] M. Sigalas, E.N. Economou, Elastic and acoustic wave band structure. J. Sound Vibr. 158, 377-382 (1992).

[8] M. Sigalas, E.N. Economou, Band structure of elastic waves in two dimensional systems. Solid State Commun. 86, 141-143 (1993).

[9] R. Martinez-Sala, J. Sancho, J.V. Sanchez-Perez, J. Llinares, F. Meseguer, Sound attenuation by sculpture. Nature 378, 241 (1997).

[10] Y.Y. Chen, Z. Ye, Acoustic attenuation by two-dimensional arrays of rigid cylinders. Phys. Rev Lett. 87, 184301184305 (2001).

[11] Y.Y. Chen, Z. Ye, Theoretical analysis of acoustic stop bands in two-dimensional periodic scattering arrays. Phys. Rev. E 64, 36616-36622 (2001).

[12] D. Caballero, J. Sánchez-Dehesa, C. Rubio, R. MártinezSala, J.V. Sánchez-Pérez, F. Meseguer, J. Llinares, Large two-dimensional sonic band gaps. Phys. Rev. E 60, R6316R6319 (1999).

[13] D. Caballero, J. Sánchez-Dehesa, R. Martínez-Sala, C. Rubio, J.V. Sánchez-Pérez, L. Sanchis, F. Meseguer, Suzuki phase in two-dimensional sonic crystals. Phys. Rev. B 64, 6430364308 (2001).

[14] L. Sanchis, F. Cervera, J. Sánchez-Dehesa, J.V. SánchezPérez, C. Rubio, R. Mártinez-Sala, Reflectance properties of two-dimensional sonic band-gaps crystals. J. Acoust. Soc. Am. 109, 2598-2605 (2001).

[15] Z. Liu, X. Zhang, Y. Mao, Y. Zhu, Z. Yang, C.T. Chan, P. Sheng, Locally Resonant Sonic Materials. Science 289, 1734-1736 (2000)

[16] M. Torres, F.R. Montero de Espinosa, J.L. Aragon, Ultrasonic wedges for elastic wave bending and splitting without requiring a full band gap. Phys. Rev. Lett. 86, 4282-4285 (2001).

[17] F. Cervera, L. Sanchis, J.V. Sanchez-Perez, R. MartinezSala, C. Rubio, F. Meseguer, C. Lopez, D. Caballero, J. Sanchez-Dehesa, Refractive acoustic devices for airborne sound. Phys. Rev. Lett. 88, 23902-23906 (2002).

[18] L. Sanchis, A. Hakansson, F. Cervera and J. SanchezDehesa, Acoustic interferometers based on two-dimensional arrays of rigid cylinders in air. Phys. Rev. B 67, 35422-135422-11 (2003).

[19] A. Khelif, P.A. Deymier, B. Djafari-Rouhani, J.O. Vasseur, L. Dobrzynski, Two-dimensional phononic crystal with tunable narrow pass band: application to a waveguide with selective frequency. J. Appl. Phys. 94, 1308-1311 (2003).

[20] B. Liang, B. Yuan, J.-C. Cheng, Acoustic diode: rectification of acoustic energy flux in one-dimensional systems. Phys. Rev. Lett. 103, 104301-104305 (2009).

[21] D.O. Cahill, W.K. Ford, K.E. Goodson, Nanoscale thermal transport. J. Appl. Phys. 93, 793 (2003).

[22] S. Murad, I.K. Puri, Thermal transport through superlattice solid-solid interfaces. Appl. Phys. Lett. 95, 051907 (2009).

[23] S.T. Huxtable, A.R. Abramson, C.-L. Tien, A. Majumdar, C. Labounty, X. Fan, G. Zeng, J.E. Bowers, A. Shakouri, Thermal conductivity of $\mathrm{Si} / \mathrm{SiGe}$ and $\mathrm{SiGe/SiGe}$ superlattices. Appl. Phys. Lett. 80, 1737-1739 (2002).

[24] J.-Y. Duquesene, Thermal conductivity of semiconductor superlattices: Experimental study of interface scattering. Phys. Rev. B 79, 153304 (2009).

[25] G. Chen, Thermal conductivity and ballistic-phonon transport in the cross-plane direction of superlattices. Phys. Rev. B 57, 14958-14973 (1998).

[26] M. Kafesaki, M.M. Sigalas, N. Garcia, The finite difference time domain method for the study of two-dimensional acoustic and elastic gap materials. Kluwer Academic Publishers, Dordrecht 2001.

[27] M. Qiu, S. He, FDTD algorithm for computing the offplane band structure in a two-dimensional photonic crystal with dielectric or metallic inclusions. Phys. Lett. A 278, 348-354 (2001).

[28] A. Taflove, Computational Electrodynamics; The FiniteDifference Time-Domain Method. Artech House, London 1995.

[29] D. Garcia-Pablos, M. Sigalas, F.R. Montero de Espinosa, M. Torres, M. Kafesaki, N. García, Theory and experiments on elastic band gaps. Phys. Rev. Lett. 84, 4349-4352 (2000).

[30] S. Mizuno, S. Tamura, Theory of acoustic-phonon transmission in finite-size superlattice systems. Phys. Rev. B 45, 734-741 (1992).

[31] J.O. Vasseur, B. Djafari-Rouhani, L. Dobrzynski, P.A. Deymier, Acoustic band gaps in fibre composite materials of boron-nitride structure. J. Phys.: Condens. Matter 9, 73277333 (1997).

[32] J.O. Vasseur, B. Djafari-Rouhani, L. Dobrzynski, M.S. Kushwaha, P. Halevi, Complete acoustic band gaps in periodic fibre reinforced composite materials: the carbon/epoxy composite and some metallic systems. J. Phys.: Condens. Matter 6, 87599-8770 (1994).

[33] GNU Scientific Library (GSL): http://www.gnu.org/software/gsl/. 


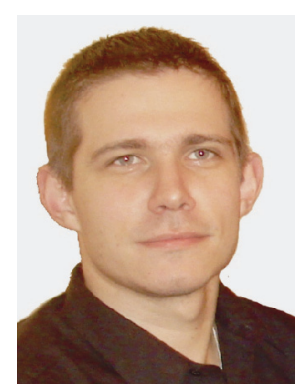

PrzemysŁaw NowaK received MSc degree in physics at the Adam Mickiewicz University, Faculty of Physics Poznan (2005). He is a PhD student at the same University and the same Faculty. His main field of interest covers applied informatics and especially programming (C++, C\#, Delphi, Java, SQL).

MACIEJ KRawCZYK received PhD degree in Faculty of Physics at Adam Mickiewicz University (2001) and he has habilitated in physics in 2009 at the same University. His field of interest covers solid state physics, dynamics in magnetic materials and metamaterials, and modeling of propagation characteristics of classical waves. 